\title{
LYMPHANGIOMATOUS MACROGLOSSIA
}

REPoRt of a CaSe

\section{John W. Kemper, D.D.S., M.D.,* and Herbert J. BloOM, D.D.S., M.D.†}

$\mathrm{M}$ ACROGLOSSIA, by definition, denotes an abnormal enlargement of the tongue. Explicitly the term covers two common forms; museular hypertrophy, and enlargement, or dilation of the lymphatic spaces. We shall confine ourselves to the commonest form-the so-called "Iymphangiomatous macroglossia.",

The true etiology is slightly clouded by conflicting opinions. Most authorities, however, agree that it is an endothelial tissue tumor composed of dilated lymphatic spaces. It is of congenital origin, often appearing at birth or shortly thereafter, although it may remain quiescent for a long period and become noticeable after dentition or before puberty, at which time it may become the seat of characteristic attacks of inflammation followed by periods of remission.

The picture of lymphangioma of the tongue is so striking and characteristic that it is worthy of mention. Minute clear vesicles appear on the surfaces of the tongue, between which are scattered capillary loops appearing as bright red dots. Many capillaries protrude into the vesicles and, because of the thinning of the walls, are prone to rupture, thereby changing the color of the vesicle to bluish black. Dilation of the lymphatic spaces is accompanied by inflammation, with the formation of dense intercystic connective tissue. There is progressive increase in the number of thin-wallen, coiled arteries and veins, and, with exacerbations, there is coincident blood extravasation into the lymphatic cavities.

With increase in size there is inability to close the mouth, and the tongue gradually protrudes beyond the oral cavity. The exposed portion of the tongue becomes dry, crustcd, fissured, and hemorrhages freely. With trauma, ulceration may occur, and with each inflammatory episode there is resultant fibrous tissue deposition and consequent further enlargement. Mastication becomes difficult, speech is blurred, and saliva dribbles from the mouth. In young patients there is distortion of the mandible in the form of depression of the horizontal rami, and if dentition is present the incisor teeth may ineline labially.

Interestingly, as in the following case, many observers report associated cervical hygromata or other forms of lymphangiomata of the face and neck. The association is curious and not clearly understood.

Therapy is confined to radiation, ignipuncture, or surgical intervention. The latter is usually carried out by removal of a wedge-shaped section or marginal resection of the tongue. A combination of all procedures may have merit in some cases.

\footnotetext{
From the Department of Oral Surgery, University Hospital, University of Michigan.

* Professor of Oral Surgery and Head of Department of Oral Surgery, Schoul of Dentistry, University Hospital, University of Michigan.

$\dagger$ Postgraduate Instructor in Oral Surgery, W. K. Kellogg Foundation Institute of Graduate and Postgraduate Dentistry.
} 


\section{CASE REPORT}

History-C. E. T., No. 516226, a 17-month-old girl, was admitted to the University Hospital in November, 1942, with the chief complaint of enlargement and bleeding of the tongue. The mother stated that the onset of illness was approximately four months prior to this date with an insidious swelling of the tongue accompanied by bleeding from its surface. The signs increased in severity and the tongue gradually protruded from the mouth, permitting the exposed surfaces to become dry and increasing the extent of the hemorrhage. The ehild apparently ate well without significant pain; however, she choked frequently during deglutition.

The patient had been a full-term baby, born of a normal delivery, and had been entirely well during the early months of her life. The familial history was noneontributory.

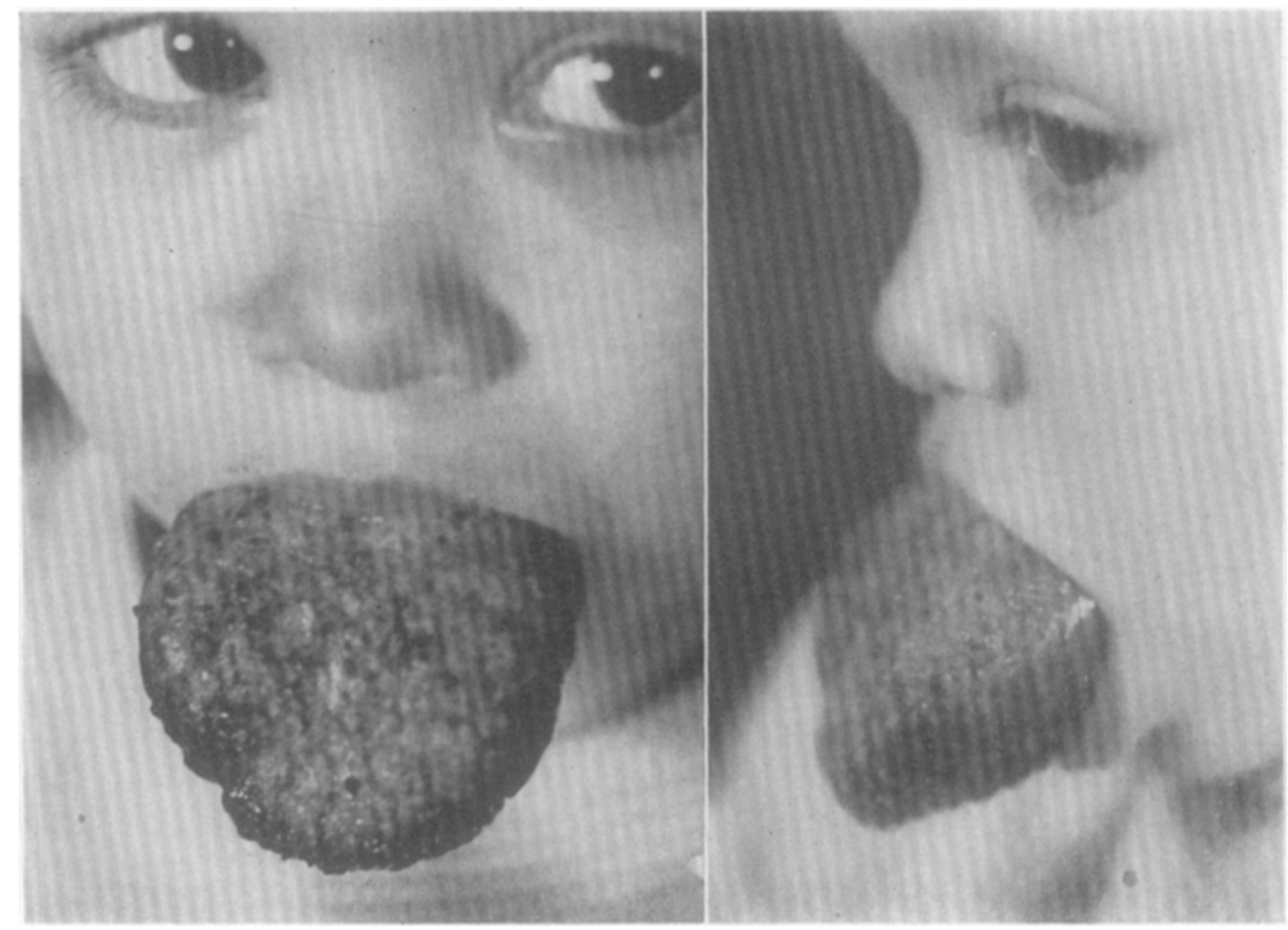

Fig. 1.- Photograph showing enormous tongue protruding from the mouth. Note small vesicles and dark nodules with crusting of blood and debris along the margins.

Examination.-The child was well developed, well nourished, but with a marked pallor, and appeared chronically ill. The gross deformity of the tongue was obvious as it appeared to be about two and a half times its normal size and protruded about $2 \mathrm{~cm}$. beyond the edges of the anterior teeth, causing the mouth to remain constantly open. The organ was firm and freely movable. Demarcation between normal and abnormal tissue could not be detected. The surface had a purplish color with scattered vesicles, and bright red, tiny elevations over its greater portion. Hemorrhagic tendency was moderate at the margins. A slight fullness was noted in the left submaxillary area, but the 
borders could not be outlined by digital examination. Further detailed examination did not disclose other evidence of significant disease.

Laboratory Data.-The blood Kahn was negative; the urine findings were normal. On admission, the hemoglobin was 55 per cent (Sahli); red blood cells $1,800,000$; white blood cells, 3,000 , with a normal differential.

Radiographic Findings.-The lungs were negative; the airway through the pharynx appeared adequate; the thymus was not enlarged. Examination of the long bones revealed normal epiphyseal development.

Course.-Following admission, the child was placed on a general supportive regime, and repeated whole blood transfusions were instituted. At the end of a week the hemoglobin had risen to 88 per cent. Under an ether anesthetic the tongue was biopsied, and the pathologist returned a diagnosis of cavernous lymphangioma with hemorrhage into the angiomatous spaces.

Radiation therapy as a form of treatment was ruled out after the radiologists declared that significant improvement could not be expected by this method. The case was then presented before a conference composed of members from the staffs of the various branches of medicine and surgery that were interested in this special problem. Representatives of the Departments of Pediatries, Otolaryngology, General Surgery, Oral Surgery, and Anatomy attended, and a discussion ensued as to the possibility of the lesion being a manifestation of lymphatic obstruetion rather than true neoplastic tissue. Partly to satisfy a few of the members of the hospital staff who favored the obstruction theory and also to examine adequately the fullness of the left side of the neck, it was agreed to explore the cervical lymphaties and to do a partial marginal glossectomy in order to create a wound with a large surface area that would, through wound healing, create a larger lymphatic bed and possibly increase lymph drainage by the proliferation of new lymph vessels.

Under ether anesthesia, a few drops of pontamine sky blue dye were first injected into the tip of the tongue to facilitate visualization and to demonstrate the lymphatics of the tongue. There was almost immediate discoloration of the complete tongue with the exception of the margins of the posterior third. A 2 inch horizontal incision was then made extending from the angle of the mandible anteriorly and $2 \mathrm{~cm}$. below the inferior border. After penetrating the superficial fascia, what appeared to be a thin-walled eyst came into view. $\Lambda$ spiration substantiated these suspicions, by obtaining a turbid fluid. There was no trace of the blue dye injected into the tongue. A radiopaque oil (lipiodol) was then injected into the cystic space. Radiograms of the neck were made immediately, and operative procedures were discontinued pending radiographic findings. The radiograms disclosed the opaque medium to be present in a large multiloculated bizarre pool extending on the left side into the floor of the mouth and upper cervical region.

Following an adequate preparatory program, the child was again returned to the operating room and the cervical wound was reopened to explore and remove the cystic lymphangiona. It was found to be a multilocular cyst extending into the submaxillary triangle and into the lateral pharyngeal space. Many thin-walled loculi containing amber-colored fluid were opened. As complete removal of the very thin-walled cysts was a surgical improbability, and because of its dissecting tendency and close adhesions to important structures 
and larger vessels, the septa were broken down to form one large eavity. This was packed loosely with a gauze dressing saturated with 5 per cent solution of sodium morrhuate. The histopathology of a tissue specimen from this area revealed it to be compatible with a diagnosis of cavernous lymphangioma or cystic cervical hygroma. The right cervical region was also explored surgically. but no cysts could be demonstrated.

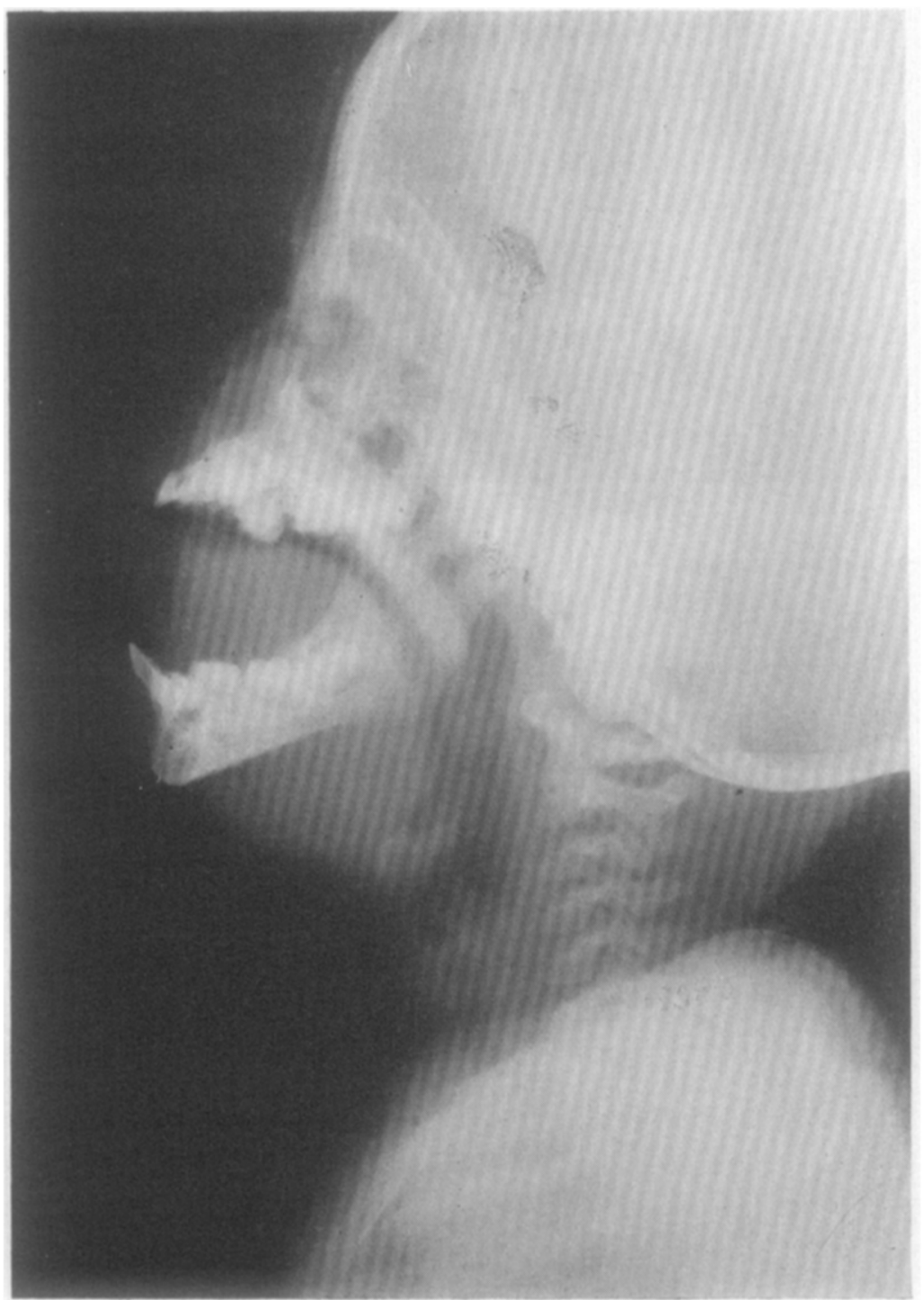

Fig. 2.- Soft tissue radiograph illustrates the enormous size of tongue. Note the labial inclination of the anterior teeth and deformity of the body of the mandible due to pressure of the tongue.

Postoperative care included changing the sodium morrhuate dressing at forty-eight-hour intervals, decreasing the size of each succeeding dressing. At the end of ten days the cystic cavity was completely obliterated. Repeated whole blood transfusions were continued to replenish the constant blood loss from the tongue. 


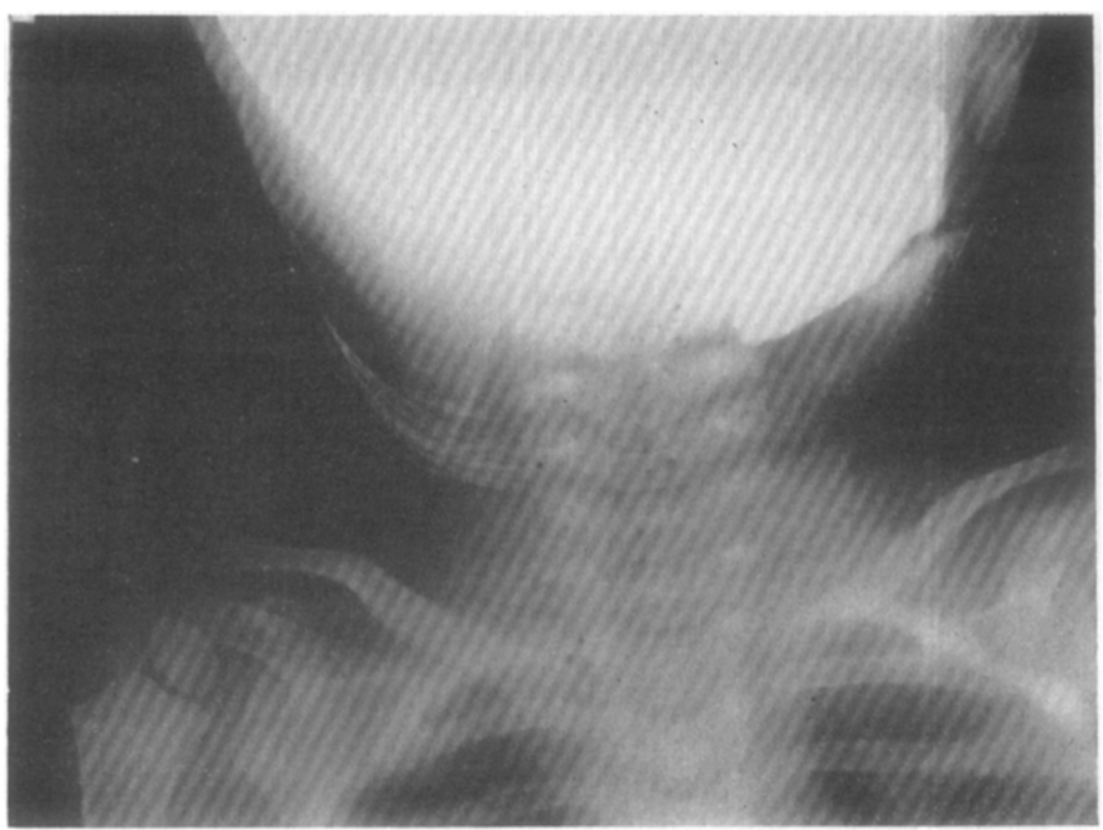

Fig. 3. •

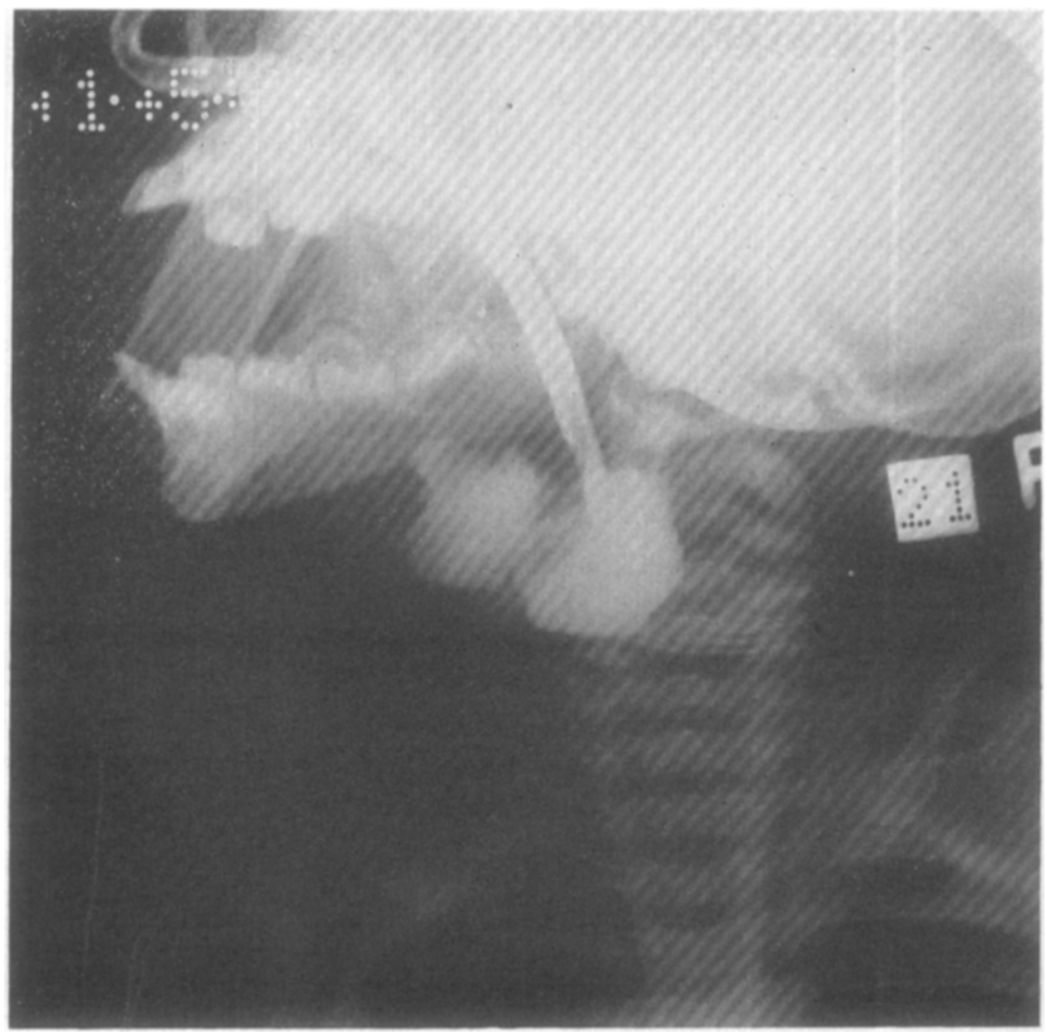

Fig. 4.

Figs. 3 and 4.-Tateral and posteroanterior radiographs showing size and position of multiloculated cyst of floor of mouth and cervical region, demonstrated by lipiodol injection. 
Two weeks following the last operation the patient was returned to the operating room, where, under ether anesthesia, a V-shaped section of tissue was removed from the margin of the tongue on either side, extending from the posterior third anteriorly through the middle third. The section measured approximately $7.5 \mathrm{~cm}$. in length and $0.5 \mathrm{~cm}$. in width and extended to the midline of the body of the tongue. It was hoped that the procedure would produce a tongue of diminished size by actual removal of tissue and that with wound healing a wider bed of lymphatic drainage would be created by proliferation of lymphatic vessels.

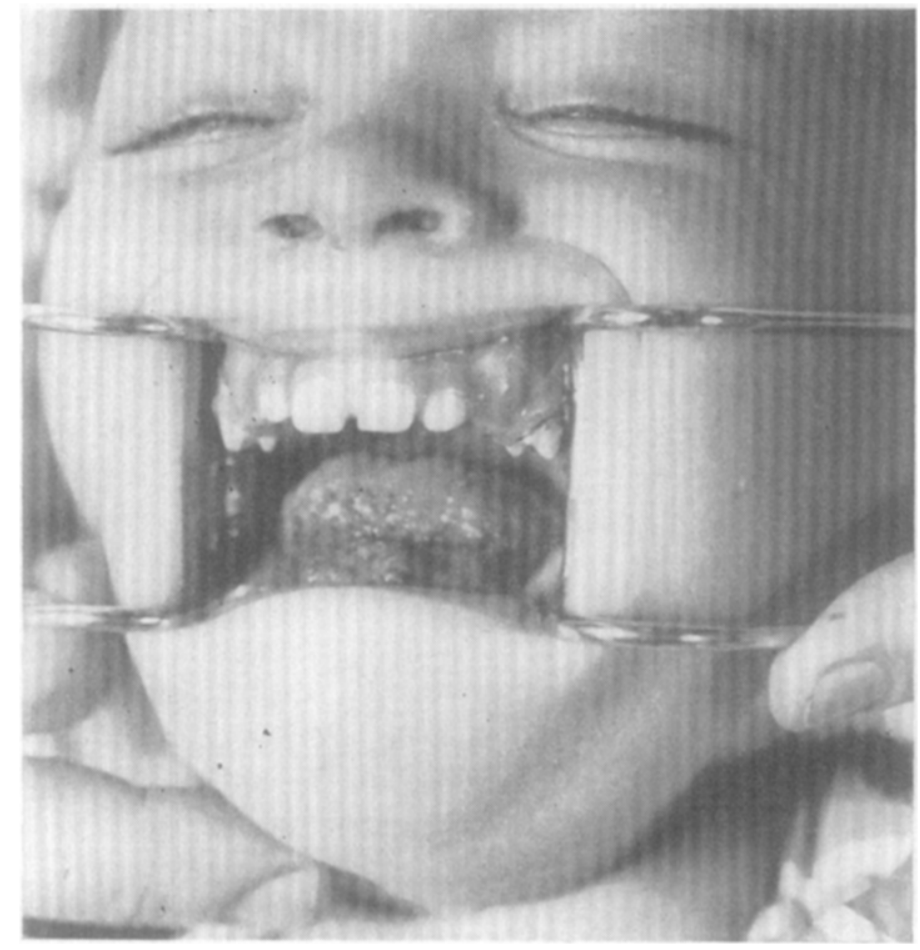

Fig. 5.-Photograph three months postoperative. The tongue is normal in size and contour and function. Along the margins can be seen some of the remaining lymphangiomatous tissue.

As the operation failed to produce the desired result, six weeks later a more extensive partial glossectomy was performed. This was carried out by making two vertical incisions through the body of the tongue, each starting about the mid-portion and directed forward and laterally. The result was the removal of a $\mathrm{V}$-shaped section from the mid-anterior region through its entire thickness. The removed tissue was about $4.5 \mathrm{~cm}$. in length including the anterior third of the tongue. The lateral sections were then approximated in the midline, producing a fairly well-shaped tongue which could be entirely contained within the oral cavity. There was no troublesome postoperative bleeding. It was noted, however, that a deformity of the mandible had been produced in the form of a depression of the anterior portion of the body of the mandible with a resultant anterior open-bite relationship of about $1 \mathrm{~cm}$. The immediate and subsequent postoperative course was uneventful, and the patient was discharged on the tenth postoperative day for an observation period. 
Six months later, E. T. was again admitted to the hospital after a completely negative interim. Oral examination revealed the tongue to be well healed, with an excellent cosmetic and functional result. There appeared to be residual neoplastic tissue over the dorsum and tip of the tongue, and a mass of lymphangiomatous tissue, about $1 \mathrm{~cm}$. in diameter, could be outlined in the floor of the mouth just lingual to the incisor teeth. The latter was removed during this admission and healing occurred. without complication.

Subsequent checkup examinations to date have not revealed significant changes; however, it is expected that further surgery of the tongue may be required.

\section{COMMENT}

Lymphangiomata commonly involve the tongue and may be single, multiple, unilateral, or bilaterally diffuse as in this case. The latter type is the rarest of the lymphangiomata of the tongue. The solitary and unilateral lesions which are well demarcated usually offer little difficulty in eradication by excision, but the bilateral diffuse type create a more complicated problem. Cavernous lymphangiomas are generally considered not an obstruction of the lymphatics but a true neoplasm and must be treated as such. The use of radium, clectrocoagulation, and partial glossectomy are the usually accepted methods of treatment. Radium and ignipuncture create more scar tissue, resulting in a more rigid organ, than surgical excision. Prognosis is generally good even though complete excision is not possible in the extensive tumors.

The lining membrane of the multilocular cervical cyst was so thin, with adhesions to the adjacent large vessels and other structures, that complete surgical removal was thought to be impossible. It was felt that by rupturing all of the loculi and creating one large cavity, the endothelial cells could be destroyed by the use of a sclerosing solution. Another similar case of a large lymphangiomatous cyst involving the floor of the mouth and extending into the base of the tongue was satisfactorily treated by us with this method. 